\title{
Assessing the segmentation performance of pairwise and triplet Markov models
}

\author{
Ivan Gorynin, Hugo Gangloff, Emmanuel Monfrini, Wojciech Pieczynski* \\ SAMOVAR, Telecom SudParis, CNRS, Université Paris-Saclay 9, rue Charles Fourier, Evry 91000, France
}

\section{A R T I C L E I N F O}

\section{Article history:}

Received 7 July 2017

Revised 4 October 2017

Accepted 4 December 2017

Available online 8 December 2017

\section{Keywords:}

Hidden Markov models

PMM

TMM

Forecasting

Financial time series

\begin{abstract}
A B S T R A C T
The hidden Markov models (HMMs) are state-space models widely applied in time series analysis. Wellknown Bayesian state estimation methods designed for HMMs, such as the Baum-Welch algorithm and the Viterbi algorithm, allow state estimation with a complexity linear in the sample size. We consider recent extensions of HMMs, specifically the pairwise Markov models (PMMs) and the triplet Markov models (TMMs), in which the Baum-Welch algorithm also has a complexity linear in the sample size. However, the state process is not necessarily Markovian in PMMs and TMMs, which offers a considerable flexibility of modeling. This study explores potential performance gains achievable if PMMs and TMMs are used to describe the state-space system rather than HMMs. This is done through extensive comparative Monte-Carlo experiments among HMMs, PMMs and TMMs in the case of discrete state space models. A simple comparative example of the use of PMMs and HMMs to predict market direction is also given. These experiments confirm the interest of PMMs and TMMs in the time series modeling: specifically, the classification rate can be improved by nearly fifty percent. These findings mean that PMMs and TMMs may be more suitable than classic HMMs for real-world applications.
\end{abstract}

(c) 2017 Elsevier B.V. All rights reserved.

\section{Introduction}

The hidden Markov model (HMM [1-3]) is an important tool in the modern modeling of various types of problems and is an active topic of research activity. This model is extensively reviewed in the literature [4-7]. Let us consider a hidden random sequence $\mathbf{X}=\left\{\mathrm{X}_{1}, \ldots, \mathrm{X}_{N}\right\}=\mathrm{X}_{1: N}$ and an observed one $\mathbf{Y}=\mathrm{Y}_{1: N}$, whose joint distribution is given by the probability density function of $(\mathbf{X}, \mathbf{Y})$ denoted by $p(\mathbf{x}, \mathbf{y})$. Each $X_{n}$ takes its value in $\Omega=\left\{\omega_{1}, \ldots, \omega_{K}\right\}$ and each $\mathrm{Y}_{n}$ takes its value in $\mathbb{R}$. The pair $(\mathbf{X}, \mathbf{Y})$ is a classic HMM if $\mathbf{X}$ is a Markov chain:

$p\left(\mathrm{x}_{1: N}\right)=p\left(\mathrm{x}_{1}\right) p\left(\mathrm{x}_{2} \mid \mathrm{x}_{1}\right) \ldots p\left(\mathrm{x}_{N} \mid \mathrm{x}_{N-1}\right)$,

and if the distribution of $\mathbf{Y}$ given $\mathbf{X}$ is of the following form:

$p\left(\mathrm{y}_{1: N} \mid \mathrm{x}_{1: N}\right)=p\left(\mathrm{y}_{1} \mid \mathrm{x}_{1}\right) \ldots p\left(\mathrm{y}_{N} \mid \mathrm{x}_{N}\right)$.

It is a known fact that (1) and (2) imply that $(\mathbf{X}, \mathbf{Y})$ is Markovian.

The pairwise Markov models (PMMs) extend HMMs by only assuming that $(\mathbf{X}, \mathbf{Y})$ is Markovian [8]. Since the hidden process $\mathbf{X}$ is not necessarily Markovian in PMMs, the latter are strictly more general than HMMs [9]. In a stationary and time-reversible PMM, $\mathbf{X}$ is Markovian only if and only if the conditional dependencies

\footnotetext{
* Corresponding author.

E-mail address: wojciech.pieczynski@telecom-sudparis.eu (W. Pieczynski).
}

in the PMM verify precise necessary and sufficient conditions [9]. The appeal of PMMs is that the classic Bayesian estimation algorithms, such as the Baum-Welch algorithm and the Viterbi algorithm apply in PMMs as well, thanks to the fact that $\mathbf{X}$ is Markovian given Y. Let us note that PMMs have been shown to be more efficient than HMMs in the context of unsupervised image segmentation [10] and computing global scores [11].

Next, the triplet Markov models (TMMs [12]) extend PMMs by adding a discrete-valued latent process $\mathbf{U}=\mathrm{U}_{1: N}$, where each $\mathrm{U}_{n}$ takes its value in a finite set $\left\{\lambda_{1}, \ldots, \lambda_{M}\right\}$. In such a model, $(\mathbf{X}, \mathbf{Y}, \mathbf{U})$ is Markovian. Despite that none of processes $\mathbf{X}, \mathbf{Y},(\mathbf{X}$, $\mathbf{U}),(\mathbf{X}, \mathbf{Y}),(\mathbf{Y}, \mathbf{U})$ is not necessarily Markovian, the Baum-Welch algorithm (but not necessarily the Viterbi algorithm) applies in TMMs [9]. Let us remark that a subclass of TMMs is shown to be efficient in image processing in [9], where it substantially outperforms HMMs. Further researches demonstrate that TMMs allow a particular semi-Markovian modeling of $\mathbf{X}$ [13], which is a valuable result since the hidden semi-Markov models are particularly wellsuited for a scope of applications [14,15]. Besides, the bivariate hidden Markov chains [16,17], which are similar to a subclass of TMMs, do also provide a framework for efficient data processing. For these reasons, we believe that researches on TMMs may have a considerable impact, especially in the field of latent variable modeling. TMMs apply in the context of signal processing $[13,18]$ and image processing [19]. 
The subject we focus on consists in exploring if using TMMs and PMMs instead of HMMs is meaningful for practical applications. This is done through simulation-based comparisons among several variants of PMMs and TMMs with respect to classic HMMs. Specifically, we consider Gaussian and gamma observation distributions in order to quantify the impact of skewness and excess kurtosis of the latter on the estimation accuracy. We also provide a simple comparative example of the use of PMMs and HMMs to predict market direction. This example demonstrates the potential value of PMMs.

The paper is organized as follows. The next section is devoted to PMMs, while the third one presents TMMs. Experiments are presented in Section 4, while the last section is devoted to conclusions and perspectives.

\section{Pairwise Markov models}

The pair $(\mathbf{X}, \mathbf{Y})$ is a pairwise Markov model (PMM) if its distribution is of the following form:

$p(\mathbf{x}, \mathbf{y})=p\left(\mathrm{x}_{1}, \mathrm{y}_{1}\right) p\left(\mathrm{x}_{2}, \mathrm{y}_{2} \mid \mathrm{x}_{1}, \mathrm{y}_{1}\right) \ldots p\left(\mathrm{x}_{N}, \mathrm{y}_{N} \mid \mathrm{x}_{N-1}, \mathrm{y}_{N-1}\right)$,

which means that $(\mathbf{X}, \mathbf{Y})$ is Markovian. Since the classic HMM distribution is

$p(\mathbf{x}, \mathbf{y})=p\left(\mathrm{x}_{1}\right) p\left(\mathrm{y}_{1} \mid \mathrm{x}_{1}\right) p\left(\mathrm{x}_{2} \mid \mathrm{x}_{1}\right) p\left(\mathrm{y}_{2} \mid \mathrm{x}_{2}\right) \ldots p\left(\mathrm{x}_{N} \mid \mathrm{x}_{N-1}\right) p\left(\mathrm{y}_{N} \mid \mathrm{x}_{N}\right)$,

and $p\left(\mathrm{x}_{n+1}, \mathrm{y}_{n+1} \mid \mathrm{x}_{n}, \mathrm{y}_{n}\right)$ from (3) can be written as

$p\left(\mathrm{x}_{n+1}, \mathrm{y}_{n+1} \mid \mathrm{x}_{n}, \mathrm{y}_{n}\right)=p\left(\mathrm{x}_{n+1} \mid \mathrm{x}_{n}, \mathrm{y}_{n}\right) p\left(\mathrm{y}_{n+1} \mid \mathrm{x}_{n+1}, \mathrm{x}_{n}, \mathrm{y}_{n}\right)$,

we see that a PMM is an HMM if and only if for each $n$ in $\{1$ : $N-1\}$,

$p\left(\mathrm{x}_{n+1} \mid \mathrm{x}_{n}, \mathrm{y}_{n}\right)=p\left(\mathrm{x}_{n+1} \mid \mathrm{x}_{n}\right) ;$

$p\left(\mathrm{y}_{n+1} \mid \mathrm{x}_{n+1}, \mathrm{x}_{n}, \mathrm{y}_{n}\right)=p\left(\mathrm{y}_{n+1} \mid \mathrm{x}_{n+1}\right)$.

This highlights the additional assumptions which are made implicitly when a real-world system is modeled by HMM whereas the same system could possibly be represented as a PMM.

Let us consider the Bayesian marginal-posterior-mode (MPM) estimator, which estimates the state vector $\mathbf{X}$ from $\mathbf{Y}$ by $\widehat{x}_{1: N}$ defined as

$\forall n \in 1: N, \widehat{x}_{n}=\underset{\omega \in \Omega}{\arg \max } p\left(\mathrm{x}_{n}=\omega \mid \mathbf{y}\right)$.

Let us briefly recall the PMM forward-backward algorithm which allows computing $p\left(\mathrm{x}_{n}=\omega \mid \mathbf{y}\right)$ for each $n$ in $\{1: N\}$ and $\omega$ in $\Omega$. Let us consider the following forward and backward probabilities, defined in a PMM by $\alpha_{n}\left(\mathrm{x}_{n}\right)=p\left(\mathrm{x}_{n}, \mathrm{y}_{1: n}\right)$ and $\beta_{n}\left(\mathrm{x}_{n}\right)=$ $p\left(\mathrm{y}_{n+1: N} \mid \mathrm{x}_{n}, \mathrm{y}_{n}\right)$. One can observe that $\alpha_{n}\left(\mathrm{x}_{n}\right)$ is defined exactly in the same way as in the well-known HMM version of the algorithm, while $\beta_{n}\left(\mathrm{x}_{n}\right)$ is slightly modified; indeed, in the HMM version it is $\beta_{n}\left(\mathrm{x}_{n}\right)=p\left(\mathrm{y}_{n+1: N} \mid \mathrm{x}_{n}\right)$. However, in both HMM and PMM, the following recursions allow computing $\alpha_{n}\left(\mathrm{x}_{n}\right)$ and $\beta_{n}\left(\mathrm{x}_{n}\right)$ for any $\mathrm{x}_{n}$ :

$\alpha_{1}\left(\mathrm{x}_{1}\right)=p\left(\mathrm{x}_{1}, \mathrm{y}_{1}\right)$

$\alpha_{1}\left(\mathrm{x}_{n+1}\right)=\sum_{\mathrm{x}_{n} \in \Omega} p\left(\mathrm{x}_{n+1}, \mathrm{y}_{n+1} \mid \mathrm{x}_{n}, \mathrm{y}_{n}\right) \alpha_{n}\left(\mathrm{x}_{n}\right) ;$

$\beta_{N}\left(\mathrm{x}_{N}\right)=1$

$\beta_{n}\left(\mathrm{x}_{n}\right)=\sum_{\mathrm{x}_{n+1} \in \Omega} p\left(\mathrm{x}_{n+1}, \mathrm{y}_{n+1} \mid \mathrm{x}_{n}, \mathrm{y}_{n}\right) \beta_{n+1}\left(\mathrm{x}_{n+1}\right)$.
Then $p\left(\mathrm{x}_{n}=\omega \mid \mathbf{y}\right)$ is computed in both HMMs and PMMs by:

$p\left(\mathrm{x}_{n}=\omega \mid \mathbf{y}\right)=\frac{\beta_{n}\left(\mathrm{x}_{n}\right) \alpha_{n}\left(\mathrm{x}_{n}\right)}{\sum_{\mathrm{x}_{n}^{*} \in \Omega} \beta_{n}\left(\mathrm{x}_{n}^{*}\right) \alpha_{n}\left(\mathrm{x}_{n}^{*}\right)}$.

The complexity of this algorithm is linear in $N$. The HMM version can be derived from (8) under conditions (6a) and (6b), in which case we have

$p\left(\mathrm{x}_{n+1}, \mathrm{y}_{n+1} \mid \mathrm{x}_{n}, \mathrm{y}_{n}\right)=p\left(\mathrm{x}_{n+1} \mid \mathrm{x}_{n}\right) p\left(\mathrm{y}_{n+1} \mid \mathrm{y}_{n}\right)$.

\subsection{Stationary time-reversible PMMs}

In what follows, we will consider stationary PMMs for which $p\left(\mathrm{x}_{n}, \mathrm{y}_{n}, \mathrm{x}_{n+1}, \mathrm{y}_{n+1}\right)$ does not depend on $n$. Thus, the whole distribution is defined by $p\left(\mathrm{x}_{1}, \mathrm{y}_{1}, \mathrm{x}_{2}, \mathrm{y}_{2}\right)$. In addition, we assume that the model is time-reversible, which means that for each $\omega_{i}, \omega_{j}$ in $\Omega$ and $\mathrm{y}, \mathrm{y}^{\prime}$ in $\mathbb{R}$,

$$
\begin{aligned}
p\left(\mathrm{x}_{1}\right. & \left.=\omega_{i}, \mathrm{y}_{1}=\mathrm{y}, \mathrm{x}_{2}=\omega_{j}, \mathrm{y}_{2}=\mathrm{y}^{\prime}\right) \\
& =p\left(\mathrm{x}_{2}=\omega_{i}, \mathrm{y}_{2}=\mathrm{y}, \mathrm{x}_{1}=\omega_{j}, \mathrm{y}_{1}=\mathrm{y}^{\prime}\right) .
\end{aligned}
$$

Let us recall the following general result shown in [9]:

Proposition 1. Let $(\mathbf{X}, \mathbf{Y})$ be a stationary time-reversible PMM. The following conditions are equivalent:

- $\mathbf{X}$ is a Markov chain;

- for each $n$ in $\{1: N-1\}, p\left(\mathrm{y}_{n+1} \mid \mathrm{x}_{n+1}, \mathrm{x}_{n}\right)=p\left(\mathrm{y}_{n+1} \mid \mathrm{x}_{n+1}\right)$;

- for each $n$ in $\{1: N\}, p\left(\mathrm{y}_{n} \mid \mathrm{x}_{1: N}\right)=p\left(\mathrm{y}_{n} \mid \mathrm{x}_{n}\right)$.

Thus, in a stationary time-reversible PMM $(\mathbf{X}, \mathbf{Y}), \mathbf{X}$ is Markovian if and only if

$p\left(\mathrm{y}_{2} \mid \mathrm{x}_{1}, \mathrm{x}_{2}\right)=p\left(\mathrm{y}_{2} \mid \mathrm{x}_{2}\right)$,

which is equivalent to

$p\left(\mathrm{y}_{1} \mid \mathrm{x}_{1}, \mathrm{x}_{2}\right)=p\left(\mathrm{y}_{1} \mid \mathrm{x}_{1}\right)$.

Let us notice that the whole distribution of a PMM can be derived from $p\left(\mathrm{x}_{1}, \mathrm{y}_{1}, \mathrm{x}_{2}, \mathrm{y}_{2}\right)$, which may be represented in the following form:

$p\left(\mathrm{x}_{1}, \mathrm{y}_{1}, \mathrm{x}_{2}, \mathrm{y}_{2}\right)=p\left(\mathrm{x}_{1}, \mathrm{x}_{2}\right) p\left(\mathrm{y}_{1}, \mathrm{y}_{2} \mid \mathrm{x}_{1}, \mathrm{x}_{2}\right)$.

Let us consider the following PMM submodels:

- "HMM-IN" which is the classic HMM of form (1) and (2). The suffix "IN" stands for "independent noise", as opposed to the "correlated noise" denoted by the suffix " $\mathrm{CN}$ ", and both terms refer to the field of image processing where PMMs were first considered for practical use. The related transition kernel $p\left(\mathrm{x}_{n+1}, \mathrm{y}_{n+1} \mid \mathrm{x}_{n}, \mathrm{y}_{n}\right)$ is

$p\left(\mathrm{x}_{2}, \mathrm{y}_{2} \mid \mathrm{x}_{1}, \mathrm{y}_{1}\right)=p\left(\mathrm{x}_{2} \mid \mathrm{x}_{1}\right) p\left(\mathrm{y}_{2} \mid \mathrm{x}_{2}\right)$.

$p\left(\mathrm{x}_{1}, \mathrm{y}_{1}, \mathrm{x}_{2}, \mathrm{y}_{2}\right)$ always verifies

$p\left(\mathrm{x}_{1}, \mathrm{y}_{1}, \mathrm{x}_{2}, \mathrm{y}_{2}\right)=p\left(\mathrm{x}_{1}, \mathrm{x}_{2}\right) p\left(\mathrm{y}_{1} \mid \mathrm{x}_{1}\right) p\left(\mathrm{y}_{2} \mid \mathrm{x}_{2}\right)$

in HMMs-IN.

- "HMM-CN", where $\mathbf{X}$ is Markovian, observation variables $\mathrm{Y}_{1: N}$ are correlated given $\mathbf{X}$, and which is not an HMM-IN (see Fig. 1). The related transition kernel is

$p\left(\mathrm{x}_{2}, \mathrm{y}_{2} \mid \mathrm{x}_{1}, \mathrm{y}_{1}\right)=p\left(\mathrm{x}_{2} \mid \mathrm{x}_{1}\right) p\left(\mathrm{y}_{2} \mid \mathrm{x}_{2}, \mathrm{y}_{1}\right)$.

- "PMM-IN", where $\mathbf{X}$ is not Markovian and observation variables $Y_{1: N}$ are independent given $X$. In PMM-IN we have $p\left(\mathrm{y}_{2} \mid \mathrm{x}_{1}, \mathrm{x}_{2}, \mathrm{y}_{1}\right)=p\left(\mathrm{y}_{2} \mid \mathrm{x}_{1}, \mathrm{x}_{2}\right)$ and

$p\left(\mathrm{x}_{2}, \mathrm{y}_{2} \mid \mathrm{x}_{1}, \mathrm{y}_{1}\right)=p\left(\mathrm{x}_{2} \mid \mathrm{x}_{1}, \mathrm{y}_{1}\right) p\left(\mathrm{y}_{2} \mid \mathrm{x}_{2}, \mathrm{x}_{1}\right)$;

$p\left(\mathrm{x}_{1}, \mathrm{y}_{1}, \mathrm{x}_{2}, \mathrm{y}_{2}\right)=p\left(\mathrm{x}_{1}, \mathrm{x}_{2}\right) p\left(\mathrm{y}_{1} \mid \mathrm{x}_{1}, \mathrm{x}_{2}\right) p\left(\mathrm{y}_{2} \mid \mathrm{x}_{1}, \mathrm{x}_{2}\right)$. 


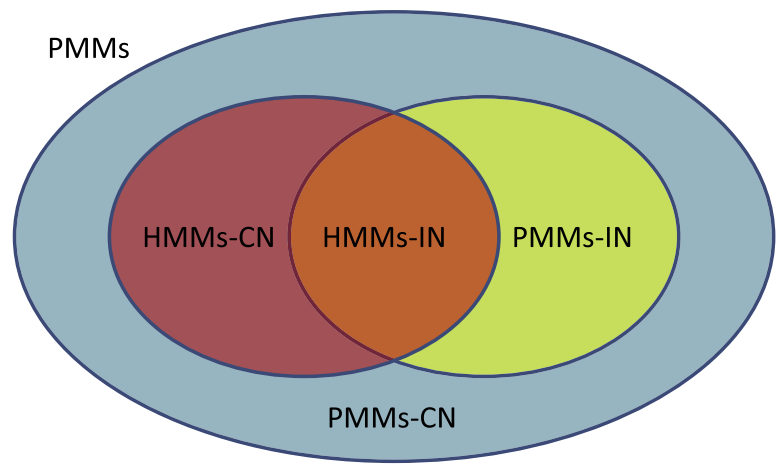

Fig. 1. Venn diagram for various PMM submodels. The area contained by all of the three circles represents PMMs. PMMs-CN are represented by the rock blue color, PMMs-IN and HMMs-CN are represented by rose and yellow respectively. The orange color represents classic HMMs-IN. (For interpretation of the references to color in this figure legend, the reader is referred to the web version of this article.)

- "PMM-CN", where $\mathbf{X}$ is not Markovian and observation variables $Y_{1: N}$ are correlated given $\mathbf{X}$, which is thus neither HMM-IN, PMM-IN nor HMM-CN (see Fig. 1). The related transition kernel is of the general form

$p\left(\mathrm{x}_{2}, \mathrm{y}_{2} \mid \mathrm{x}_{1}, \mathrm{y}_{1}\right)=p\left(\mathrm{x}_{2} \mid \mathrm{x}_{1}, \mathrm{y}_{1}\right) p\left(\mathrm{y}_{2} \mid \mathrm{x}_{2}, \mathrm{x}_{1}, \mathrm{y}_{1}\right)$.

The dependency graphs of the four PMM submodels are presented in Fig. 2.

\section{Triplet Markov models}

Let us consider sequences $\mathbf{X}$ and $\mathbf{Y}$ as previously. The triplet Markov model (TMM) makes use of an additional discrete-valued process $\mathbf{U}=\mathrm{U}_{1: N}$, where each $\mathrm{U}_{n}$ takes its value in a finite set $\Lambda=\left\{\lambda_{1}, \ldots, \lambda_{M}\right\}$. By definition, the triplet $(\mathbf{X}, \mathbf{Y}, \mathbf{U})$ is Markovian in TMMs.

The TMM framework allows a similar MPM estimation of $\mathbf{X}$ from $\mathbf{Y}$ to that of HMMs and PMMs. It is feasible thanks to the fact that $(\mathbf{V}, \mathbf{Y})$ is a PMM, where $\mathbf{V}=(\mathbf{X}, \mathbf{U})$. It allows computing $p\left(\mathrm{x}_{n}, \mathrm{u}_{n} \mid \mathrm{y}_{1: N}\right)$ and then

$p\left(\mathrm{x}_{n} \mid \mathrm{y}_{1: N}\right)=\sum_{\mathrm{u}_{n} \in \Lambda} p\left(\mathrm{x}_{n}, \mathrm{u}_{n} \mid \mathrm{y}_{1: N}\right)$.

We notice that it is possible to efficiently compute the Bayesian-optimal MPM estimates of $\mathbf{X}$ despite the fact that (X, $\mathbf{Y})$ is not Markovian of in TMMs. An example of such setting is presented in Section 4.3. Besides, TMMs have a high potential of modeling; specifically, $\mathbf{U}$ can be multivariate in a way that each sequence $\mathbf{U}^{(i)}$ in $\mathbf{U}=\left[\mathbf{U}^{(1)} ; \ldots ; \mathbf{U}^{(s)}\right]^{\top}$ would represent a separate property. For example, some non-stationary hidden semiMarkov models can be seen as a TMM $\left(\mathbf{X}, \mathbf{Y}, \mathbf{U}^{(1)}, \mathbf{U}^{(2)}\right)$ in which $\mathbf{U}^{(1)}$ models the semi-Markovianity and $\mathbf{U}^{(2)}$ stands for the nonstationarity [13].

In the previous section, we consider three extensions of the classic HMMs-IN. Here we propose two other ones, which are
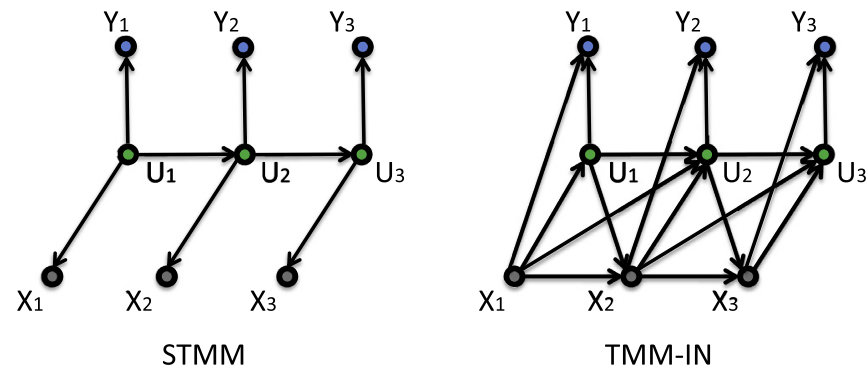

Fig. 3. Dependency graphs of STMM and TMM-IN

based on the TMM: the simplified TMM (STMM) and the TMM with independent noise (TMM-IN).

- STMM is a stationary time-reversible TMM, whose distribution is defined by

$$
\begin{aligned}
& p\left(\mathrm{x}_{1}, \mathrm{u}_{1}, \mathrm{y}_{1}, \mathrm{x}_{2}, \mathrm{u}_{2}, \mathrm{y}_{2}\right) \\
& \quad=p\left(\mathrm{u}_{1}, \mathrm{u}_{2}\right) p\left(\mathrm{y}_{1} \mid \mathrm{u}_{1}\right) p\left(\mathrm{x}_{1} \mid \mathrm{u}_{1}\right) p\left(\mathrm{y}_{2} \mid \mathrm{u}_{2}\right) p\left(\mathrm{x}_{2} \mid \mathrm{u}_{2}\right) .
\end{aligned}
$$

The corresponding transition kernel is

$p\left(\mathrm{x}_{2}, \mathrm{u}_{2}, \mathrm{y}_{2} \mid \mathrm{x}_{1}, \mathrm{u}_{1}, \mathrm{y}_{1}\right)=p\left(\mathrm{u}_{2} \mid \mathrm{u}_{1}\right) p\left(\mathrm{y}_{2} \mid \mathrm{u}_{2}\right) p\left(\mathrm{x}_{2} \mid \mathrm{u}_{2}\right)$.

$(\mathbf{X}, \mathbf{Y})$ is not Markovian in STMM. Thus, STMM is different from PMM. In fact, one can see an STMM as a hidden Markov model with $\mathbf{U}$ hidden and ( $\mathbf{X}, \mathbf{Y})$ observed, and it is well-known that the observed process is not Markovian in such a model.

- TMM-IN is an extension of the STMM on the one hand, and an extension of the classic HMM-IN on the other hand. Specifically, let $\mathbf{V}=(\mathbf{X}, \mathbf{U})$, then $(\mathbf{V}, \mathbf{Y})$ is a classic HMM-IN and this is why we denote it by TMM-IN. Thus, the distribution of a stationary TMMIN is given by

$p\left(\mathrm{x}_{1}, \mathrm{u}_{1}, \mathrm{y}_{1}, \mathrm{x}_{2}, \mathrm{u}_{2}, \mathrm{y}_{2}\right)=p\left(\mathrm{u}_{1}, \mathrm{u}_{2}, \mathrm{x}_{1}, \mathrm{x}_{2}\right) p\left(\mathrm{y}_{1} \mid \mathrm{u}_{1}, \mathrm{x}_{1}\right) p\left(\mathrm{y}_{2} \mid \mathrm{u}_{2}, \mathrm{x}_{2}\right)$

and the corresponding transition kernel is

$p\left(\mathrm{x}_{2}, \mathrm{u}_{2}, \mathrm{y}_{2} \mid \mathrm{x}_{1}, \mathrm{u}_{1}, \mathrm{y}_{1}\right)=p\left(\mathrm{u}_{2}, \mathrm{x}_{2} \mid \mathrm{u}_{1}, \mathrm{x}_{1}\right) p\left(\mathrm{y}_{2} \mid \mathrm{u}_{2}, \mathrm{x}_{2}\right)$.

The dependency graphs of STMM and TMM-IN are given in Fig. 3.

\section{Experiments}

\subsection{Gaussian pairwise Markov models}

We present different experiments comparing PMMs-CN, PMMsIN, HMMs-CN and HMMs-IN from Section 2. We set $\Omega=\left\{\omega_{1}, \omega_{2}\right\}$ for the sake of simplicity.

We parameterize PMM-CN by $\epsilon \in[0,0.5]$ and $\rho \in[0,1]$ as follows:

$p\left(\mathrm{x}_{1}, \mathrm{x}_{2}\right)= \begin{cases}0.5-\epsilon & \text { if } \mathrm{x}_{1}=\mathrm{x}_{2} \\ \epsilon & \text { if } \mathrm{x}_{1} \neq \mathrm{x}_{2}\end{cases}$

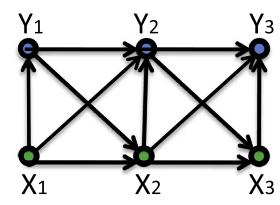

PMM-CN

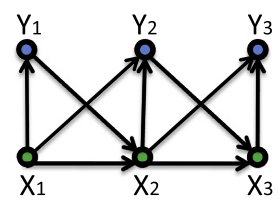

PMM-IN

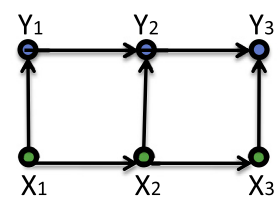

HMM-CN

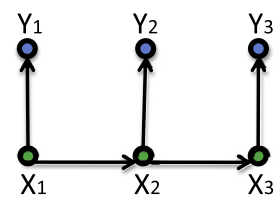

HMM-IN

Fig. 2. Dependency graphs of PMM-CN, PMM-IN, HMM-CN and HMM-IN. 
Table 1

Mean and variance parameters of Gaussian distributions (25b).

\begin{tabular}{lllll}
\hline$\left(\mathrm{x}_{1}, \mathrm{x}_{2}\right)$ & $\mu_{1}\left(\mathrm{x}_{1}, \mathrm{x}_{2}\right)$ & $\mu_{2}\left(\mathrm{x}_{1}, \mathrm{x}_{2}\right)$ & $\sigma_{1}\left(\mathrm{x}_{1}, \mathrm{x}_{2}\right)$ & $\sigma_{2}\left(\mathrm{x}_{1}, \mathrm{x}_{2}\right)$ \\
\hline$\left(\omega_{1}, \omega_{1}\right)$ & -5 & -5 & 14 & 14 \\
$\left(\omega_{1}, \omega_{2}\right)$ & -3 & 3 & 7 & 9 \\
$\left(\omega_{2}, \omega_{1}\right)$ & 3 & -3 & 9 & 7 \\
$\left(\omega_{2}, \omega_{2}\right)$ & 5 & 5 & 20 & 20 \\
\hline
\end{tabular}

$$
\begin{aligned}
& p\left(\mathrm{y}_{1}, \mathrm{y}_{2} \mid \mathrm{x}_{1}, \mathrm{x}_{2}\right)=\mathscr{N}\left(\left[\begin{array}{l}
\mu_{1}\left(\mathrm{x}_{1}, \mathrm{x}_{2}\right) \\
\mu_{2}\left(\mathrm{x}_{1}, \mathrm{x}_{2}\right)
\end{array}\right],\right. \\
& \left.\left[\begin{array}{cc}
\sigma_{1}^{2}\left(\mathrm{x}_{1}, \mathrm{x}_{2}\right) & \rho \sigma_{1}\left(\mathrm{x}_{1}, \mathrm{x}_{2}\right) \sigma_{2}\left(\mathrm{x}_{1}, \mathrm{x}_{2}\right) \\
\rho \sigma_{1}\left(\mathrm{x}_{1}, \mathrm{x}_{2}\right) \sigma_{2}\left(\mathrm{x}_{1}, \mathrm{x}_{2}\right) & \sigma_{2}^{2}\left(\mathrm{x}_{1}, \mathrm{x}_{2}\right)
\end{array}\right]\right) .
\end{aligned}
$$

In the above equation, $\mathscr{N}(\mathbf{m}, \boldsymbol{\Sigma})$ is the Gaussian distribution with mean vector $\mathbf{m}$ and variance-covariance matrix $\boldsymbol{\Sigma}$. The coefficients $\epsilon$ and $\rho$ depend on the experimental setting; the values of the remaining parameters per each pair $\left(\mathrm{x}_{1}, \mathrm{x}_{2}\right)$ are fixed and presented in Table 1.

\subsubsection{Sampling in PMMs-CN}

Let us first specify the sampling procedure corresponding to Gaussian PMM-CN (25). We begin by sampling $\left(\mathrm{x}_{1}, \mathrm{x}_{2}\right)$ from (25a), then we sample $\left(\mathrm{y}_{1}, \mathrm{y}_{2}\right)$ given $\left(\mathrm{x}_{1}, \mathrm{x}_{2}\right)$ from $(25 \mathrm{~b})$. Next, given $\left(\mathrm{x}_{n}\right.$, $\left.\mathrm{y}_{n}\right)$ for $n \geq 2$, we sample $\left(\mathrm{x}_{n+1}, \mathrm{y}_{n+1}\right)$ as follows. Firstly, we sample $\mathrm{x}_{n+1}$ from $p\left(\mathrm{x}_{n+1} \mid \mathrm{x}_{n}, \mathrm{y}_{n}\right)$, where

$p\left(\mathrm{x}_{n+1} \mid \mathrm{x}_{n}, \mathrm{y}_{n}\right)=\frac{p\left(\mathrm{x}_{n}, \mathrm{x}_{n+1}\right) p\left(\mathrm{y}_{n} \mid \mathrm{x}_{n}, \mathrm{x}_{n+1}\right)}{\sum_{\mathrm{x}_{n+1}^{*} \in \Omega} p\left(\mathrm{x}_{n}, \mathrm{x}_{n+1}^{*}\right) p\left(\mathrm{y}_{n} \mid \mathrm{x}_{n}, \mathrm{x}_{n+1}^{*}\right)}$

with

$p\left(\mathrm{y}_{n} \mid \mathrm{x}_{n}, \mathrm{x}_{n+1}\right)=\mathscr{N}\left(\mu_{1}\left(\mathrm{x}_{n}, \mathrm{x}_{n+1}\right), \sigma_{1}^{2}\left(\mathrm{x}_{n}, \mathrm{x}_{n+1}\right)\right)$.

Secondly, we sample $\mathrm{y}_{n+1}$ from $p\left(\mathrm{y}_{n+1} \mid \mathrm{x}_{n}, \mathrm{x}_{n+1}, \mathrm{y}_{n}\right)$, where

$$
\begin{aligned}
& p\left(\mathrm{y}_{n+1} \mid \mathrm{x}_{n}, \mathrm{x}_{n+1}, \mathrm{y}_{n}\right) \\
& \quad=\mathscr{N}\left(\mu_{2}\left(\mathrm{x}_{n}, \mathrm{x}_{n+1}\right)+\rho \frac{\sigma_{2}\left(\mathrm{x}_{n}, \mathrm{x}_{n+1}\right)}{\sigma_{1}\left(\mathrm{x}_{n}, \mathrm{x}_{n+1}\right)}\left(\mathrm{y}_{n}-\mu_{1}\left(\mathrm{x}_{n}, \mathrm{x}_{n+1}\right)\right),\right. \\
& \left.\quad \sigma_{2}^{2}\left(\mathrm{x}_{n}, \mathrm{x}_{n+1}\right)\left(1-\rho^{2}\right)\right) .
\end{aligned}
$$

\subsubsection{HMM-IN, HMM-CN and PMM-IN approximation of PMM-CN}

The experimental setting consists in sampling $\left(\mathrm{x}_{1}, \mathrm{y}_{1}, \ldots, \mathrm{x}_{N}, \mathrm{y}_{N}\right)$ from a given PMM-CN (25), then estimating $\mathrm{x}_{1: N}$ from $\mathrm{y}_{1: N}$ by four MPM estimators corresponding to the original PMM-CN and its approximations which are PMM-IN, HMM-CN and HMM-IN. We define the related parameters as follows.

- In a Gaussian HMM-IN, one has $p\left(\mathrm{y}_{1} \mid \mathrm{x}_{1}, \mathrm{x}_{2}\right)=p\left(\mathrm{y}_{1} \mid \mathrm{x}_{1}\right)$ and $p\left(\mathrm{y}_{2} \mid \mathrm{x}_{1}, \mathrm{x}_{2}, \mathrm{y}_{1}\right)=p\left(\mathrm{y}_{2} \mid \mathrm{x}_{2}\right)$, thus

$p\left(\mathrm{y}_{1} \mid \mathrm{x}_{1}\right)=\mathscr{N}\left(\mu_{1}^{\text {(HMM-IN) }}\left(\mathrm{x}_{1}\right), \sigma_{1}^{2(\mathrm{HMM}-\mathrm{IN})}\left(\mathrm{x}_{1}\right)\right)$;

$p\left(\mathrm{y}_{2} \mid \mathrm{x}_{2}\right)=\mathscr{N}\left(\mu_{2}^{\text {(HMM-IN) }}\left(\mathrm{x}_{2}\right), \sigma_{2}^{2 \text { (HMM-IN) }}\left(\mathrm{x}_{2}\right)\right)$.

Given a Gaussian PMM-CN, we define the parameters of the corresponding HMM-IN by the principle of moment-matching:

$$
\begin{aligned}
& \mu_{1}^{\text {(HMM-IN) }}\left(\mathrm{x}_{1}\right)=\sum_{\mathrm{x}_{2} \in \Omega} \mu_{1}\left(\mathrm{x}_{1}, \mathrm{x}_{2}\right) p\left(\mathrm{x}_{2} \mid \mathrm{x}_{1}\right) ; \\
& \sigma_{1}^{2 \text { (НMM-IN) }}\left(\mathrm{x}_{1}\right) \\
& \quad=\sum_{\mathrm{x}_{2} \in \Omega}\left(\sigma_{1}^{2}\left(\mathrm{x}_{1}, \mathrm{x}_{2}\right)+\left(\mu_{1}\left(\mathrm{x}_{1}, \mathrm{x}_{2}\right)-\mu_{1}^{\text {(HMM-IN) }}\left(\mathrm{x}_{1}\right)\right)^{2}\right) p\left(\mathrm{x}_{2} \mid \mathrm{x}_{1}\right) .
\end{aligned}
$$

By the stationarity assumption, we have for any $\omega$ in $\Omega$,

$\mu_{2}^{\text {(HMM-IN) }}(\omega)=\mu_{1}^{\text {(HMM-IN) }}(\omega)$;

$\sigma_{2}^{2(\text { HMM-IN) }}(\omega)=\sigma_{1}^{2(\text { HMM-IN) }}(\omega)$.

- In a Gaussian HMM-CN, one has $p\left(\mathrm{y}_{1} \mid \mathrm{x}_{1}, \mathrm{x}_{2}\right)=p\left(\mathrm{y}_{1} \mid \mathrm{x}_{1}\right)$ and $p\left(\mathrm{y}_{2} \mid \mathrm{x}_{1}, \mathrm{x}_{2}, \mathrm{y}_{1}\right)=p\left(\mathrm{y}_{2} \mid \mathrm{x}_{2}, \mathrm{y}_{1}\right)$. Thus, we consider the same distribution $p\left(\mathrm{y}_{1} \mid \mathrm{x}_{1}\right)$ as in the case of HMM-IN defined by (31). Regarding $p\left(\mathrm{y}_{2} \mid \mathrm{x}_{2}, \mathrm{y}_{1}\right)$, we have

$$
\begin{aligned}
& p\left(\mathrm{y}_{2} \mid \mathrm{x}_{2}, \mathrm{y}_{1}\right) \\
& =\mathscr{N}\left(\mu_{1}^{\text {(HMM-IN) }}\left(\mathrm{x}_{2}\right)+\rho \frac{\sigma_{2}^{\text {(HMM-IN })}\left(\mathrm{x}_{2}\right)}{\sigma_{1 \mid 2}^{\text {(HMM-CN })}\left(\mathrm{x}_{2}\right)}\left(\mathrm{y}_{1}-\mu_{1 \mid 2}^{\text {(HMM-CN) }}\left(\mathrm{x}_{2}\right)\right),\right. \\
& \left.\sigma_{2}^{2 \text { (HMM-IN) }}\left(\mathrm{X}_{2}\right)\left(1-\rho^{2}\right)\right),
\end{aligned}
$$

where $\left\{\mu_{1 \mid 2}^{\text {(HMM-CN) }}\left(\mathrm{x}_{2}\right), \sigma_{1 \mid 2}^{\text {(HMM-CN)}}\left(\mathrm{x}_{2}\right)\right\}_{\mathrm{X}_{2} \in \Omega}$ are computed by using the principle of moment matching:

$$
\begin{aligned}
& \mu_{1 \mid 2}^{\text {(НMM-CN) }}\left(\mathrm{x}_{2}\right)=\sum_{\mathrm{x}_{1} \in \Omega} \mu_{1}\left(\mathrm{x}_{1}, \mathrm{x}_{2}\right) p\left(\mathrm{x}_{1} \mid \mathrm{x}_{2}\right) ; \\
& \sigma_{1 \mid 2}^{2 \text { (HМM-CN) }}\left(\mathrm{x}_{2}\right) \\
& =\sum_{\mathrm{x}_{1} \in \Omega}\left(\sigma_{1}^{2}\left(\mathrm{x}_{1}, \mathrm{x}_{2}\right)+\left(\mu_{1}\left(\mathrm{x}_{1}, \mathrm{x}_{2}\right)-\mu_{1 \mid 2}^{\text {(HMM-CN })}\left(\mathrm{x}_{2}\right)\right)^{2}\right) p\left(\mathrm{x}_{1} \mid \mathrm{x}_{2}\right) .
\end{aligned}
$$

- Finally, a PMM-IN approximation of PMM-CN verifies

$p\left(\mathrm{y}_{1} \mid \mathrm{x}_{1}, \mathrm{x}_{2}\right)=\mathscr{N}\left(\mu_{1}\left(\mathrm{x}_{1}, \mathrm{x}_{2}\right), \sigma_{1}^{2}\left(\mathrm{x}_{1}, \mathrm{x}_{2}\right)\right) ;$

$p\left(\mathrm{y}_{2} \mid \mathrm{x}_{1}, \mathrm{x}_{2}\right)=\mathscr{N}\left(\mu_{2}\left(\mathrm{x}_{1}, \mathrm{x}_{2}\right), \sigma_{2}^{2}\left(\mathrm{x}_{1}, \mathrm{x}_{2}\right)\right)$,

since $p\left(\mathrm{y}_{2} \mid \mathrm{x}_{1}, \mathrm{x}_{2}, \mathrm{y}_{1}\right)=p\left(\mathrm{y}_{2} \mid \mathrm{x}_{1}, \mathrm{x}_{2}\right)$, which is equivalent to assume $\rho=0$.

\subsubsection{Experiments on synthetic data}

The PMM-CN-based MPM estimator is statistically optimal in terms of the classification rate and we consider its accuracy as a reference. The aim of the experiments is to study if the misclassification rate is sensitive to the choice of approximation PMM-IN, HMM-CN or HMM-IN, and up to which extent. We apprehend this sensitivity thought the relative error rate, defined as follows:

$\tau^{\text {(model) }}=\frac{L\left(\mathbf{x}, \widehat{\mathbf{x}}^{\text {(model })}\right)-L\left(\mathbf{x}, \widehat{\mathbf{x}}^{\text {(PMM-CN })}\right)}{L\left(\mathbf{x}, \widehat{\mathbf{x}}^{\text {(PMM-CN })}\right)} ;$

$L\left(\mathbf{x}, \widehat{\mathbf{x}}^{\text {(model) })}\right)=\frac{1}{N} \sum_{n=1}^{N} \delta\left(\widehat{\mathrm{x}}_{n}^{\text {(model) }} \neq \mathrm{x}_{n}\right)$,

where $\delta($.$) is the indicator function and \widehat{\mathbf{x}}^{\text {(model) }}$ is the state estimate computed by using the Bayesian-optimal MPM state estimator related to the corresponding model. For example, a relative error rate of $100 \%$ means that the reference model decreases the misclassification percentage by a half when compared to the proposal one. We report in Tables 3 and 4 relative error rates for various values of $\epsilon$ and $\rho$. We also report in Table 2 the corresponding statistically optimal loss function values. That is to illustrate that the chosen parameters actually represent a considerable noise 
Table 2

Error rate (36b) of the reference PMM$\mathrm{CN}(25)$ for varying $\epsilon$ and $\rho$. Sample size is 1000 and the results are averaged over 100 experiments.

\begin{tabular}{lllll}
\hline$\epsilon \backslash \rho$ & 0.00 & 0.35 & 0.70 & 0.90 \\
\hline 0.05 & 0.20 & 0.24 & 0.25 & 0.24 \\
0.15 & 0.28 & 0.29 & 0.27 & 0.23 \\
0.20 & 0.29 & 0.29 & 0.25 & 0.21 \\
0.35 & 0.26 & 0.23 & 0.17 & 0.12
\end{tabular}

Table 3

Relative error rates (36a) of the three Gaussian PMM submodels for varying $\epsilon$ with $\rho=0.75$. Sample size is 1000 and the results are averaged over 100 experiments.

\begin{tabular}{llll}
\hline$\epsilon$ & HMM-IN & HMM-CN & PMM-IN \\
\hline 0.05 & $41 \%$ & $13 \%$ & $38 \%$ \\
0.15 & $47 \%$ & $34 \%$ & $25 \%$ \\
0.20 & $56 \%$ & $38 \%$ & $24 \%$ \\
0.35 & $58 \%$ & $31 \%$ & $37 \%$ \\
Avg & $\mathbf{5 1 \%}$ & $\mathbf{2 9} \%$ & $\mathbf{3 1 \%}$
\end{tabular}

Table 4

Relative error rates (36a) of the three Gaussian PMM submodels for varying $\rho$ with $\epsilon=0.125$. Sample size is 1000 and the results are averaged over 100 experiments.

\begin{tabular}{llll}
\hline$\rho$ & HMM-IN & HMM-CN & PMM-IN \\
\hline 0.00 & $13 \%$ & $13 \%$ & $0 \%$ \\
0.35 & $18 \%$ & $14 \%$ & $6 \%$ \\
0.70 & $38 \%$ & $26 \%$ & $23 \%$ \\
0.90 & $69 \%$ & $52 \%$ & $44 \%$ \\
Avg & $\mathbf{3 5 \%}$ & $\mathbf{2 6} \%$ & $\mathbf{1 8 \%}$ \\
\hline
\end{tabular}

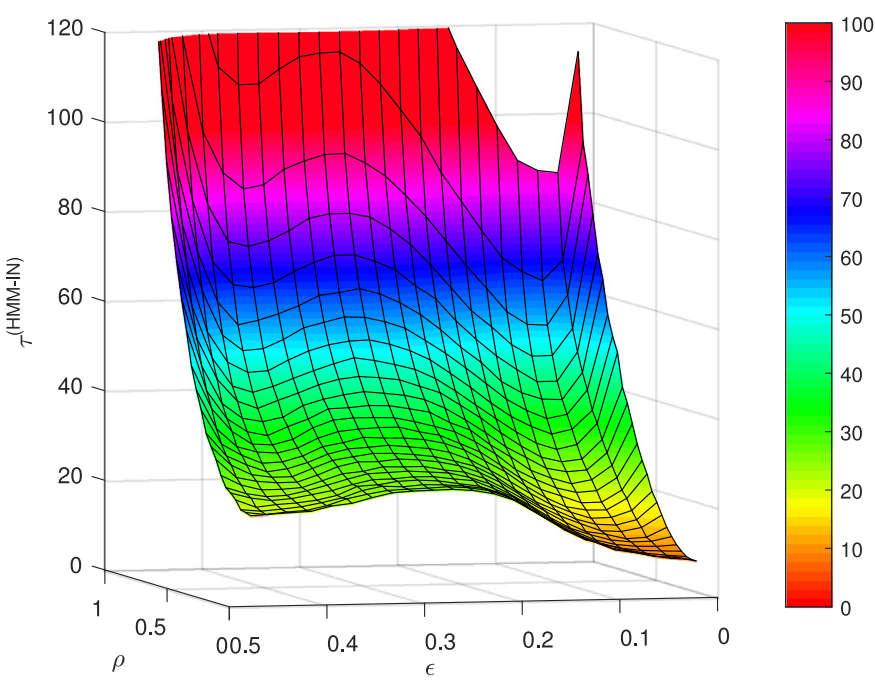

Fig. 4. Relative error rate surface plot for Gaussian HMM-IN (36a) in function of $(\epsilon$, $\rho$ ). Sample size is 1000 and the results are averaged over 100 experiments.

level. Figs. 4 and 5 present more exhaustive results regarding the relative error rate of the HMM-IN.

Regarding results presented in Tables 3 and 4, we notice that the HMM-IN approximation is the least accurate, while PMM-IN and HMM-CN have both fairly the same degree of performance. Regarding Figs. 4 and 5, we observe that $\tau^{\text {(нмм-ІN) }}<20 \%$ only if $\rho<0.4$ and $\epsilon<0.15, \tau^{\text {(НMM-IN) }}<50 \%$ only if $\rho<0.6$, and $\tau^{\text {(HMM-IN) }}<$ $80 \%$ only if $\rho<0.85$. For extreme values of $\epsilon$ in a neighborhood of 0.5 and for $\rho$ in a neighborhood of $1, \tau^{\text {(нМм-IN) }}$ diverges.

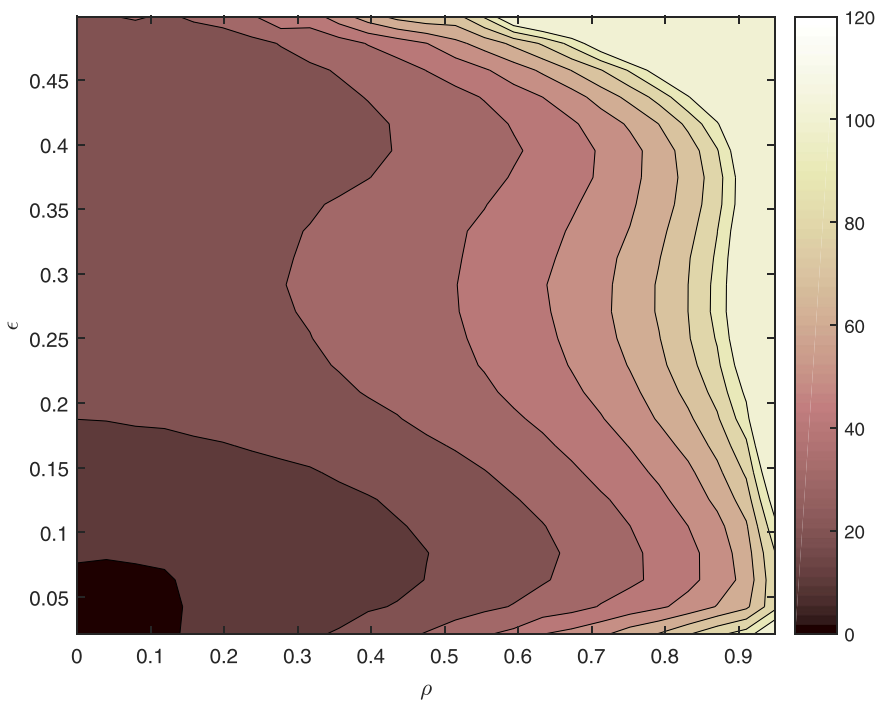

Fig. 5. Relative error rate (36a) contour plot for Gaussian HMM-IN in function of $(\epsilon, \rho)$. The contour lines refer to relative error rates of $10 \%, 20 \%, \ldots, 90 \%$ and $100 \%$. Sample size is 1000 and the results are averaged over 100 experiments.

As a discussion, we notice that both features of PMMs-CN, i.e. $p\left(\mathrm{x}_{n+1} \mid \mathrm{x}_{n}, \mathrm{y}_{n}\right) \neq p\left(\mathrm{x}_{n+1} \mid \mathrm{x}_{n}\right)$ and $p\left(\mathrm{y}_{n+1} \mid \mathrm{x}_{n}, \mathrm{y}_{n}\right) \neq p\left(\mathrm{y}_{n+1} \mid \mathrm{x}_{n+1}, \mathrm{x}_{n}\right)$ contribute independently to improving its accuracy over the simpler models. For these reasons, PMM-CN may decrease the misclassification rate of HMM-IN by a half in several settings.

\subsubsection{PMMs for market prediction}

Here we compare PMM submodels from the point of view of their capacity to fit the historical stock quotes. Specifically, we show that PMMs-CN achieve better forecasting performance than the other PMM submodels which are HMMs-IN, HMMs-CN and PMMs-IN. We consider three time series corresponding to the daily returns computed on S\&P 500, FTSE 100 and Alerian master limited partnership indices.

- ^ GSPC denotes the Standard \& Poor's 500 index (S\&P 500), which is an American stock market index based on the market capitalizations of 500 large companies having common stock listed on the NYSE or NASDAQ.

- ${ }^{\wedge}$ FTSE denotes the Financial Times Stock Exchange 100 Index, often abbreviated as FTSE 100, which is a share index of the 100 companies listed on the London Stock Exchange with the highest market capitalization.

$-{ }^{\wedge}$ AMZ denotes the Alerian master limited partnership (MLP) index, which is used by investors for performance benchmarking.

Let us present our methodology to evaluate the capacity of the four PMM submodels to fit historical stock quotes. For each stock index considered, we compute the corresponding daily log-returns $\left\{\mathrm{y}_{1}, \ldots, \mathrm{y}_{N}\right\}$ by taking the logarithm of daily returns at consecutive business days in a given period. Next, we estimate the parameters of each PMM submodel from these log-returns by the Iterative Conditional Estimation algorithm (ICE, [10]). For each stock index and PMM submodel considered, we proceed as follows. Define two-class categorical variables $\left\{Z_{n, h}\right\}_{n, h \in \mathbb{N}^{*}}$ by

$\forall(n, h) \in \mathbb{N}^{*}, Z_{n, h}= \begin{cases}1 & \text { if } \sum_{i=1}^{h} \mathrm{Y}_{n+i}>0 ; \\ 0 & \text { otherwise. }\end{cases}$

$Z_{n+h}$ represents the future market direction for the next $h$ days which follow the $n$th day. The anticipated market direction for these days given the information available at the $n$th day is de- 
Table 5

MCC values (39) relative to comparison of model-estimated market directions with the historically observed ones at horizon $h=1$. The stock data corresponds to the period from $01 / 01 / 2013$ to $01 / 01 / 2016$.

\begin{tabular}{lllll}
\hline & HMM-IN & HMM-CN & PMM-IN & PMM-CN \\
\hline$\wedge$ GSPC & 0.16 & 0.18 & 0.18 & 0.18 \\
$\wedge$ FTSE & 0.17 & 0.21 & 0.17 & 0.22 \\
$\wedge$ AMZ & 0.15 & 0.22 & 0.20 & 0.22 \\
\hline
\end{tabular}

Table 6

MCC values (39) relative to comparison of model-estimated market directions with the historically observed ones at horizon $h=5$. The stock data corresponds to the period from $01 / 01 / 2013$ to $01 / 01 / 2016$

\begin{tabular}{lllll}
\hline & HMM-IN & HMM-CN & PMM-IN & PMM-CN \\
\hline${ }^{\wedge}$ GSPC & 0.08 & 0.10 & 0.08 & 0.14 \\
$\wedge^{\wedge}$ FTSE & 0.07 & 0.14 & 0.12 & 0.15 \\
$\wedge^{\wedge}$ AMZ & 0.00 & 0.13 & 0.05 & 0.16 \\
\hline
\end{tabular}

fined by

$S_{n, h}=\left\{\begin{array}{cc}1 & \text { if } \mathbb{E}\left[Z_{n, h} \mid Y_{1 . . n}\right]>0.5 ; \\ 0 & \text { otherwise. }\end{array}\right.$

Given $h$ in $\mathbb{N}^{*}$, we use HMM-IN, HMM-CN, PMM-IN and PMMCN to compute $S_{n, h}$ at each $n$ in $\{1: N-h\}$ by Monte Carlo simulation and we compare the obtained sequence with the corresponding historical values by using the Matthews correlation coefficient (MCC, [20]). In machine learning, MCC is generally regarded as a correlation coefficient between the observed and predicted binary classifications. It ranges from -1 to 1 . An MCC coefficient close to 1 represents a perfect prediction, a value within $[-0.1,0.1]$ indicates that the prediction method considered is not better than a random guess, an MCC value close to -1 indicates total disagreement between prediction and observation. The MCC is defined as follows:

$\mathrm{MCC}=\frac{T_{P} T_{N}-F_{P} F_{N}}{\sqrt{\left(T_{P}+F_{P}\right)\left(T_{P}+F_{N}\right)\left(T_{N}+F_{P}\right)\left(T_{N}+F_{N}\right)}}$,

where $T_{P}, T_{N}, F_{P}, F_{N}$ are the number of respectively true positives, true negatives, false positives and false negatives. Specifically, they are defined as

$T_{P}=\sum_{n=1}^{N-h} \delta\left(S_{n, h}=1\right) \delta\left(Z_{n, h}=1\right) ;$

$T_{N}=\sum_{n=1}^{N-h} \delta\left(S_{n, h}=0\right) \delta\left(Z_{n, h}=0\right) ;$

$F_{P}=\sum_{n=1}^{N-h} \delta\left(S_{n, h}=1\right) \delta\left(Z_{n, h}=0\right)$

$F_{N}=\sum_{n=1}^{N-h} \delta\left(S_{n, h}=0\right) \delta\left(Z_{n, h}=1\right)$.

We apply this methodology to ${ }^{\wedge} \mathrm{GSPC}$, ${ }^{\wedge} \mathrm{FTSE}$ and ${ }^{\wedge} \mathrm{AMZ}$ quotes. These were taken from the Yahoo! database and correspond to the business days from $01 / 01 / 2013$ to $01 / 01 / 2016$. In this way, all the three data sets have the same size which is $N=783$. In this study, the state space consists of only two elements. MCC values relative to comparison of model-estimated market directions with the historically observed ones for each PMM submodel and each stock index considered are given in Table 5 for $h=1$ and Table 6 for

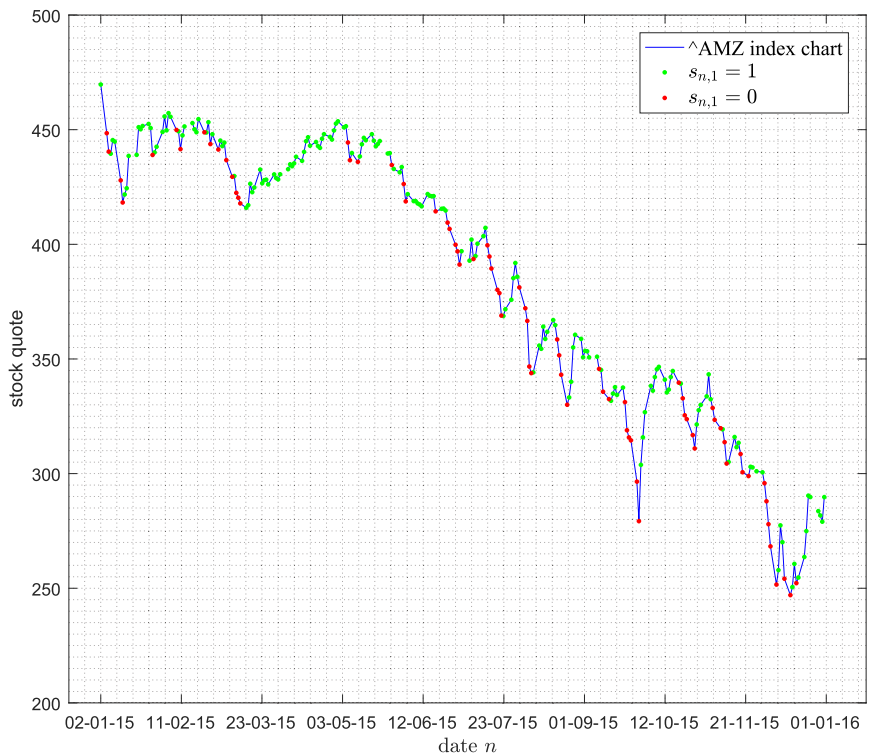

Fig. 6. ${ }^{\wedge} \mathrm{AMZ}$ directions, estimated with the $\mathrm{PMM}-\mathrm{CN}$ at each business day from $01 / 01 / 2015$ to $01 / 01 / 2016$. Green and red points indicate a predicted movement upwards and downwards respectively. (For interpretation of the references to color in this figure legend, the reader is referred to the web version of this article.)

$h=5$. Fig. 6 illustrates an example of anticipating the direction of the ${ }^{\wedge} \mathrm{AMZ}$ by PMM-CN.

Let us make several brief observations.

- By comparing the MCC values given in Table 5 for $h=1$ with the corresponding MCC values given in Table 6 for $h=5$, we see that estimating the market direction for a long horizon is more challenging than that for a short one, what is not surprising. This holds true independently of the model and stock index considered.

- Results from both Tables 5 and 6 confirm that PMM-CN has the best capacity to fit a data series as the most inclusive model across the models considered. As expected, HMM-IN has the most limited fitting capacity, while HMM-CN and PMM-IN are confirmed to be two different and independent generalizations of HMM-IN.

- We see from Table 6 that HMM-CN, PMM-IN and PMM-CN allowed to attain a statistically significant relation between the model-estimated stock direction and historically observed one at horizon $h=5$, as opposed to the HMM-IN. This illustrates that PMMs are capable of capturing a number of important features of financial time series. See [21] for a detailed discussion on an application of PMMs for market prediction.

\subsection{Gamma pairwise Markov models}

In this section we introduce a class of non-Gaussian pairwise Markov models in order to study whether previous findings generalize to a broader class of observation distributions. We consider hidden Markov models with exponential noise [22] and we extend them to hidden Markov models with gamma noise. Then we introduce PMM-CN with gamma noise and we conduct similar experiments as in the previous subsection.

For shape parameter $k$ in $\mathbb{R}_{+}^{*}$ and scale parameter $\theta$ in $\mathbb{R}_{+}^{*}$, let us note with $\gamma(k, \theta)$ the corresponding gamma distribution. Its probability density function is

$\gamma(y ; k, \theta)=\frac{y^{k-1} \exp \left(-\frac{y}{\theta}\right)}{\Gamma(k) \theta^{k}} \delta(y>0)$,

where $\delta($.$) is the indicator function and \Gamma$ is the gamma function:

$\Gamma(k)=\int_{0}^{+\infty} t^{k-1} \exp (-t) \mathrm{d} t$. 
Table 7

Error rate (36b) of gamma PMM (46) for varying $\epsilon$ and $\rho$. Sample size is 1000 and the results are averaged over 100 experiments.

\begin{tabular}{lllll}
\hline$\epsilon \backslash \rho$ & 0.00 & 0.35 & 0.70 & 0.90 \\
\hline 0.05 & 0.34 & 0.38 & 0.40 & 0.38 \\
0.15 & 0.41 & 0.43 & 0.42 & 0.39 \\
0.20 & 0.43 & 0.43 & 0.42 & 0.38 \\
0.35 & 0.43 & 0.42 & 0.38 & 0.31 \\
\hline
\end{tabular}

The exponential distribution

$\mathscr{E}(y ; k, \lambda)=\lambda \exp (-\lambda y) \delta(y>0)$

is a special case of the gamma distribution, corresponding to $k=1$ and $\theta=\frac{1}{\lambda}$. For large values of $k, \gamma(k, \theta)$ is well approximated by Gaussian distribution $\mathscr{N}\left(k \theta, k \theta^{2}\right)$; if $k$ is close to zero, the gamma distribution is highly asymmetric.

Let $\rho \in[0,1]$, we consider stationary gamma-autoregressive process [23] defined by

$p\left(\mathrm{y}_{1}\right)=\gamma\left(\mathrm{y}_{1} ; k, \theta\right)$;

$p\left(\mathrm{y}_{n+1} \mid \mathrm{y}_{n}\right)=\bar{\gamma}\left(\mathrm{y}_{n+1} ; k, \frac{\rho}{\theta(1-\rho)} \mathrm{y}_{n}, \theta(1-\rho)\right)$,

where, for $\beta$ in $\mathbb{R}_{+}^{*}, \bar{\gamma}(y ; k, \beta, \theta)$ is non-central gamma distribution

$\bar{\gamma}(y ; k, \beta, \theta)=\sum_{t=0}^{+\infty} \frac{\beta^{t} y^{k+t-1} \exp \left(-\frac{y}{\theta}\right)}{t ! \Gamma(k+t) \theta^{k+t} \exp (\beta)} \delta(y>0)$.

The mean and variance of $\bar{\gamma}(k, \beta, \theta)$ are $k \theta+\beta \theta$ and $k \theta^{2}+$ $2 \beta \theta^{2}$ respectively; besides, we have $\bar{\gamma}(k, 0, \theta)=\gamma(k, \theta)$.

Hence, let $\sigma_{1}, \sigma_{2}: \Omega^{2} \rightarrow \mathbb{R}_{+}^{*}, \mu_{1}, \mu_{2}: \Omega^{2} \rightarrow \mathbb{R}, \rho \in[0,1]$, we define gamma PMM-CN as follows:

$p\left(\mathrm{y}_{1} \mid \mathrm{x}_{1}, \mathrm{x}_{2}\right)=\gamma\left(\mathrm{y}_{1}-\mu_{1}\left(\mathrm{x}_{1}, \mathrm{x}_{2}\right) ; k, \theta_{1}\left(\mathrm{x}_{1}, \mathrm{x}_{2}\right)\right) ;$

$$
\begin{aligned}
p\left(\mathrm{y}_{n+1} \mid \mathrm{y}_{n}, \mathrm{x}_{n}, \mathrm{x}_{n+1}\right) & \\
= & \bar{\gamma}\left(\mathrm{y}_{n+1}-\mu_{2}\left(\mathrm{x}_{1}, \mathrm{x}_{2}\right) ; k, \frac{\rho\left(\mathrm{y}_{n}-\mu_{1}\left(\mathrm{x}_{1}, \mathrm{x}_{2}\right)\right)}{\theta_{1}\left(\mathrm{x}_{n}, \mathrm{x}_{n+1}\right)(1-\rho)},\right. \\
& \left.\theta_{2}\left(\mathrm{x}_{n}, \mathrm{x}_{n+1}\right)(1-\rho)\right),
\end{aligned}
$$

where $\theta_{1}\left(\mathrm{x}_{n}, \mathrm{x}_{n+1}\right)=\frac{\sigma_{1}\left(\mathrm{x}_{n}, \mathrm{x}_{n+1}\right)}{\sqrt{k}}, \theta_{2}\left(\mathrm{x}_{n}, \mathrm{x}_{n+1}\right)=\frac{\sigma_{2}\left(\mathrm{x}_{n}, \mathrm{x}_{n+1}\right)}{\sqrt{k}}$.

This model is consistent with the definition of the autoregressive gamma process in the same way as the Gaussian PMM is consistent with the autoregressive Gaussian process. Moreover, this model generalizes exponential hidden Markov models: in gamma HMMs, $\sigma_{1}\left(\mathrm{x}_{n}, \mathrm{x}_{n+1}\right), \mu_{1}\left(\mathrm{x}_{n}, \mathrm{x}_{n+1}\right)$ depend only on $\mathrm{x}_{n}$, $\sigma_{2}\left(\mathrm{x}_{n}, \mathrm{x}_{n+1}\right), \mu_{2}\left(\mathrm{x}_{n}, \mathrm{x}_{n+1}\right)$ depend only on $\mathrm{x}_{n+1}$ and $\rho=0$; exponential HMMs verify additionally $k=1$. Any gamma PMM can be approximated by a gamma HMM-IN, gamma HMM-CN and gamma PMM-IN by using the corresponding formulas (31), (32), (34) and (35).

Similarly to the previous subsection, we report in Tables 8 and 9 relative error rates of the three submodels of gamma PMM and we report in Table 7 the corresponding statistically optimal loss function values. We consider the case of exponential models i.e. $k=1$. The values of $\sigma_{1}\left(\mathrm{x}_{n}, \mathrm{x}_{n+1}\right), \mu_{1}\left(\mathrm{x}_{n}, \mathrm{x}_{n+1}\right), \sigma_{2}\left(\mathrm{x}_{n}, \mathrm{x}_{n+1}\right), \mu_{2}\left(\mathrm{x}_{n}, \mathrm{x}_{n+1}\right)$ are the same as previously and given in Table 1 .

This simulation study shows that non-Gaussian PMMs allow achieving substantial gains in accuracy, as well as the Gaussian ones. Moreover, PMMs seem outperform HMMs even more when
Table 8

Relative error rates (36a) of the three gamma PMM submodels for varying $\epsilon$ and $\rho=0.75$, $k=1$. Sample size is 1000 and the results are averaged over 100 experiments.

\begin{tabular}{llll}
\hline$\epsilon$ & HMM-IN & HMM-CN & PMM-IN \\
\hline 0.05 & $79 \%$ & $75 \%$ & $16 \%$ \\
0.15 & $130 \%$ & $115 \%$ & $23 \%$ \\
0.20 & $171 \%$ & $125 \%$ & $30 \%$ \\
0.35 & $186 \%$ & $125 \%$ & $63 \%$ \\
Avg & $\mathbf{1 4 2 \%}$ & $\mathbf{2 9 \%}$ & $\mathbf{3 3} \%$ \\
\hline
\end{tabular}

Table 9

Relative error rates (36a) of the three gamma PMM submodels for varying $\rho$ and $\epsilon=0.125$, $k=1$. Sample size is 1000 and the results are averaged over 100 experiments.

\begin{tabular}{llll}
\hline$\rho$ & HMM-IN & HMM-CN & PMM-IN \\
\hline 0.00 & $118 \%$ & $118 \%$ & $0 \%$ \\
0.35 & $114 \%$ & $112 \%$ & $4 \%$ \\
0.70 & $113 \%$ & $109 \%$ & $17 \%$ \\
0.90 & $129 \%$ & $108 \%$ & $41 \%$ \\
Avg & $\mathbf{1 1 9} \%$ & $\mathbf{1 1 2} \%$ & $\mathbf{1 6} \%$ \\
\hline
\end{tabular}

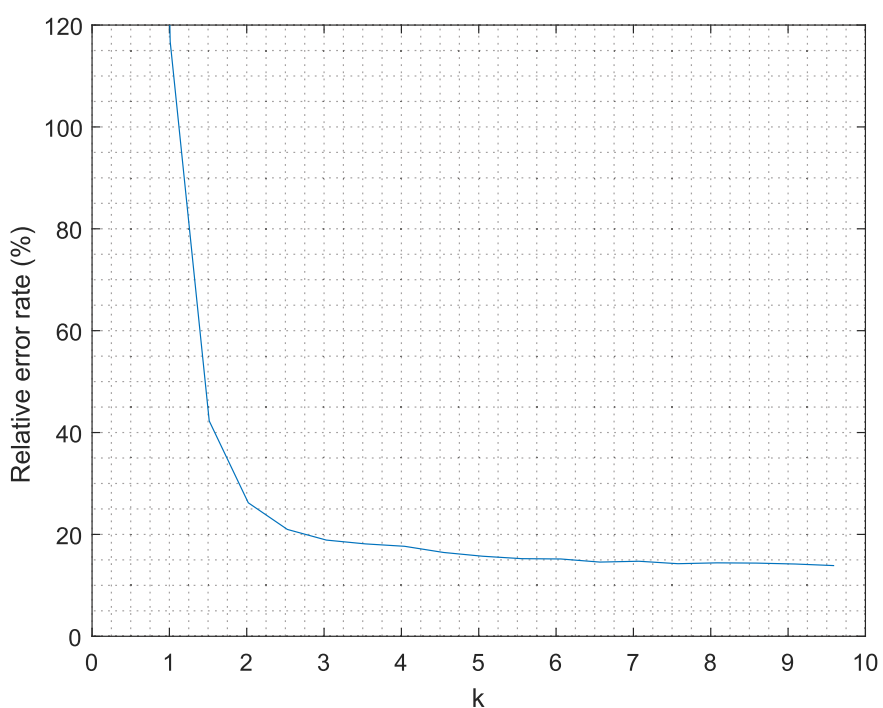

Fig. 7. Relative error rate (36a) of gamma HMM-IN with respect to gamma PMM$\mathrm{CN}$, for $\epsilon=0.125$ and $\rho=0$, in function of the shape parameter $k$. Sample size is 1000 and the results are averaged over 100 experiments.

the observation distributions are asymmetric. In order to validate this finding, we consider a fixed pair $(\epsilon, \rho)$ and we gradually increase the value of the shape parameter $k$ from 1 to 10 . We report the corresponding relative error rates of gamma HMM-IN with respect to gamma PMM-CN in Fig. 7. When $k=1$, the corresponding relative error rate is $118 \%$ and can be found in Table 9. When $k=10$, the corresponding gamma distribution is close to the normal distribution, and the corresponding relative error rate can be found in Table 4.

\subsection{STMM compared to the HMM-IN}

In this subsection we compare the STMM from Section 3 with the classic HMM-IN. Specifically, we simulate data according to an STMM and we recover $\mathbf{X}$ from $\mathbf{Y}$ with the STMM on the one hand, and with an HMM-IN on the other hand. 


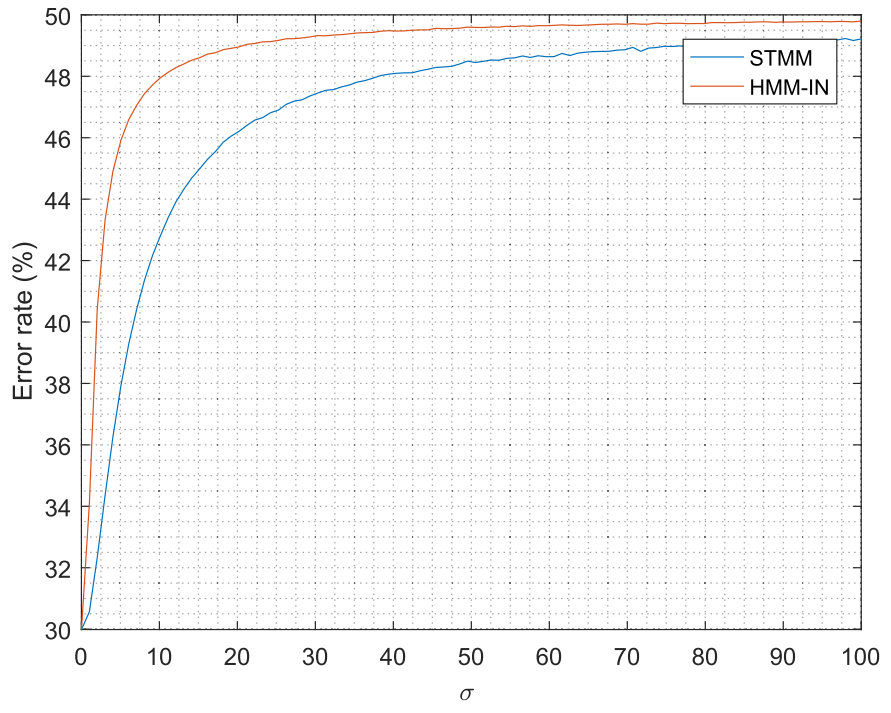

Fig. 8. Misclassification rates of STMM and HMM-IN for various values of $\sigma$ in (47c). Sample size is 1000 and the results are averaged over 100 experiments.

Let $\Omega=\left\{\omega_{1}, \omega_{2}\right\}$ and $\Lambda=\left\{\lambda_{1}, \lambda_{2}\right\}$, we define the following STMM, whose distribution (21) is specified as follows:

$p\left(\mathrm{u}_{1}, \mathrm{u}_{2}\right)= \begin{cases}0.49 & \text { if } \mathrm{u}_{1}=\mathrm{u}_{2} \\ 0.01 & \text { if } \mathrm{u}_{1} \neq \mathrm{u}_{2}\end{cases}$

$p\left(\mathrm{x}_{1} \mid \mathrm{u}_{1}\right)= \begin{cases}0.7 & \text { if } \mathrm{x}_{1}=\mathrm{u}_{1} \\ 0.3 & \text { if } \mathrm{x}_{1} \neq \mathrm{u}_{1}\end{cases}$

$p\left(\mathrm{y}_{1} \mid \mathrm{u}_{1}\right)=\mathscr{N}\left(\mu_{\mathrm{u}}\left(\mathrm{u}_{1}\right), \sigma^{2}\right)$,

where $\mu_{\mathrm{u}}\left(\lambda_{1}\right)=-1$ and $\mu_{\mathrm{u}}\left(\lambda_{2}\right)=1$. We define the distribution $p\left(\mathrm{x}_{1}, \mathrm{y}_{1}, \mathrm{x}_{2}, \mathrm{y}_{2}\right)$ in (15) of the HMM-IN approximation to (21) as follows:

$p\left(\mathrm{x}_{1}, \mathrm{x}_{2}\right)=\sum_{\mathrm{u}_{1}, \mathrm{u}_{2} \in \Lambda} p\left(\mathrm{u}_{1}, \mathrm{u}_{2}\right) p\left(\mathrm{x}_{1} \mid \mathrm{u}_{1}\right) p\left(\mathrm{x}_{2} \mid \mathrm{u}_{2}\right) ;$

$p\left(\mathrm{y}_{1} \mid \mathrm{x}_{1}\right)=\mathscr{N}\left(\mu_{\mathrm{x}}\left(\mathrm{x}_{1}\right), \sigma_{\mathrm{x}}^{2}\left(\mathrm{x}_{1}\right)\right)$,

where the parameters of $p\left(\mathrm{y}_{1} \mid \mathrm{x}_{1}\right)$ are computed by the principle of moment-matching:

$\mu_{\mathrm{x}}\left(\mathrm{x}_{1}\right)=\sum_{\mathrm{u}_{1} \in \Lambda} p\left(\mathrm{u}_{1} \mid \mathrm{x}_{1}\right) \mu_{\mathrm{u}}\left(\mathrm{u}_{1}\right)$

$\sigma_{\mathrm{x}}^{2}\left(\mathrm{x}_{1}\right)=\sigma^{2}+\sum_{\mathrm{u}_{1} \in \Lambda}\left(\mu_{\mathrm{u}}\left(\mathrm{u}_{1}\right)-\mu_{\mathrm{x}}\left(\mathrm{x}_{1}\right)\right)^{2} p\left(\mathrm{u}_{1} \mid \mathrm{x}_{1}\right)$.

The experiment reported in Fig. 8 consists in computing misclassification rates for STMM and its HMM-IN approximation for various values of $\sigma$. We observe that HMM-IN approximation appears to be fairly suboptimal for several values of $\sigma$.

\subsection{TMM-IN compared to the HMM-IN and its three extensions}

In this subsection we compare TMM-IN (23) from Section 3 with the classic HMM-IN and its three extensions. The first one is known as the mixture-HMM [24] and is a classic hidden Markov model where $\mathbf{X}$ is Markovian, observation variables $Y_{1: N}$ are independent given $\mathbf{X}$ and the observation density is represented by a mixture of Gaussian distributions. We denote it as mixture-HMM-IN. The two others are obtained from HMM-IN and mixture-HMM-IN by considering Markovianity of order 2, $c f$.
Table 10

Probability values $\left\{p\left(\mathrm{x}_{1}, \mathrm{u}_{1}, \mathrm{x}_{2}, \mathrm{u}_{2}\right)\right\}$ for $\mathrm{x}_{1}, \mathrm{x}_{2}$ in $\Omega$ and $\mathrm{u}_{1}, \mathrm{u}_{2}$ in $\Lambda$.

\begin{tabular}{lllll}
\hline$\left(\mathrm{x}_{1}, \mathrm{u}_{1}\right)\left(\mathrm{x}_{2}, \mathrm{u}_{2}\right)$ & $\left(\omega_{1}, \lambda_{1}\right)$ & $\left(\omega_{1}, \lambda_{2}\right)$ & $\left(\omega_{2}, \lambda_{1}\right)$ & $\left(\omega_{2}, \lambda_{2}\right)$ \\
\hline$\left(\omega_{1}, \lambda_{1}\right)$ & 0.22 & 0.01 & 0.01 & 0.01 \\
$\left(\omega_{1}, \lambda_{2}\right)$ & 0.01 & 0.22 & 0.01 & 0.01 \\
$\left(\omega_{2}, \lambda_{1}\right)$ & 0.01 & 0.01 & 0.22 & 0.01 \\
$\left(\omega_{2}, \lambda_{2}\right)$ & 0.01 & 0.01 & 0.01 & 0.22 \\
\hline
\end{tabular}

e.g. [7]. They are denoted by HMM-IN-2 and mixture-HMM-IN-2 respectively. We set $\Omega=\left\{\omega_{1}, \omega_{2}\right\}$ and $\Lambda=\left\{\lambda_{1}, \lambda_{2}\right\}$.

- The mixture-HMM-IN can be identified as a TMM-IN submodel in which $\mathbf{X}$ and $\mathbf{U}$ are independent and variables $U_{1: N}$ are independent too. The corresponding distribution of $(\mathbf{X}, \mathbf{U}, \mathbf{Y})$ is the following:

$$
\begin{aligned}
p(\mathbf{x}, \mathbf{u}, \mathbf{y})= & p\left(\mathrm{x}_{1}\right) p\left(\mathrm{x}_{2} \mid \mathrm{x}_{1}\right) \ldots p\left(\mathrm{x}_{N} \mid \mathrm{x}_{N-1}\right) p\left(\mathrm{u}_{1}\right) p\left(\mathrm{u}_{2}\right) \ldots p\left(\mathrm{u}_{N}\right) \\
& \times p\left(\mathrm{y}_{1} \mid \mathrm{x}_{1}, \mathrm{u}_{1}\right) \ldots p\left(\mathrm{y}_{N} \mid \mathrm{x}_{N}, \mathrm{u}_{N}\right) .
\end{aligned}
$$

- In an HMM-IN-2, one has

$$
\begin{aligned}
p(\mathbf{x}, \mathbf{y})= & p\left(\mathrm{x}_{1}, \mathrm{x}_{2}\right) p\left(\mathrm{x}_{3} \mid \mathrm{x}_{1}, \mathrm{x}_{2}\right) \ldots p\left(\mathrm{x}_{N} \mid \mathrm{x}_{N-1}, \mathrm{x}_{N-2}\right) \\
& \times p\left(\mathrm{y}_{1} \mid \mathrm{x}_{1}\right) \ldots p\left(\mathrm{y}_{N} \mid \mathrm{x}_{N}\right) .
\end{aligned}
$$

Thus, an HMM-IN-2 is technically a TMM-IN where $\Lambda=\Omega$ and for each $n$ in $\{1: N\}, \mathrm{U}_{n}=\mathrm{X}_{n-1}$ and $p\left(\mathrm{y}_{n} \mid \mathrm{x}_{n}, \mathrm{u}_{n}\right)=p\left(\mathrm{y}_{n} \mid \mathrm{x}_{n}\right)$. Note that if $K$ is the number of elements in $\Omega, K^{\prime}$ is that of $\Lambda$, then the dimension of the hidden space of TMM is $K K^{\prime}$. Thus, HMM-IN-2 is equivalent to TMM-IN in terms of the dimension of the hidden space if $K=K^{\prime}$. Therefore, we find meaningful to compare TMMsIN with HMMs-IN as well as with HMMs-IN-2 in our experiments.

The state distribution $p\left(\mathrm{x}_{1}, \mathrm{u}_{1}, \mathrm{x}_{2}, \mathrm{u}_{2}\right)$ within TMMs-IN considered for the experiments is given in Table 10 .

Regarding the observation space, we set

$p\left(\mathrm{y}_{1} \mid \mathrm{u}_{1}, \mathrm{x}_{1}\right)=\mathscr{N}\left(\mu\left(\mathrm{u}_{1}, \mathrm{x}_{1}\right), 1\right)$.

Let us consider three following cases of positioning of $\mu\left(\mathrm{u}_{1}, \mathrm{x}_{1}\right)$ :

1. Case 1: $\mu\left(\omega_{1}, \lambda_{1}\right)<\mu\left(\omega_{1}, \lambda_{2}\right)<\mu\left(\omega_{2}, \lambda_{2}\right)<\mu\left(\omega_{2}, \lambda_{1}\right)$;

2. Case 1: $\mu\left(\omega_{1}, \lambda_{1}\right)<\mu\left(\omega_{2}, \lambda_{1}\right)<\mu\left(\omega_{2}, \lambda_{2}\right)<\mu\left(\omega_{1}, \lambda_{2}\right)$;

3. Case 1: $\mu\left(\omega_{1}, \lambda_{1}\right)<\mu\left(\omega_{2}, \lambda_{1}\right)<\mu\left(\omega_{1}, \lambda_{2}\right)<\mu\left(\omega_{2}, \lambda_{2}\right)$.

Given the symmetries of $p\left(\mathrm{x}_{1}, \mathrm{u}_{1}, \mathrm{x}_{2}, \mathrm{u}_{2}\right)$, these cases are exhaustive regarding the problem of estimation of $\mathbf{X}$ from $\mathbf{Y}$. We consider sampling $(\mathbf{X}, \mathbf{Y})$ from TMM-IN and estimating $\mathbf{X}$ by HMMIN, HMM-IN-2, mixture-HMM-IN, mixture-HMM-IN-2 and TMM-IN estimators. In our experiments, we consider simulated samples of size 1000 and we average results over 100 independent identical experiments.

In Case 1, all the five estimators yield pretty much the same result.

In Case 2, we consider $\Delta>0$ and set

$$
\left\{\begin{array}{l}
\mu\left(\omega_{1}, \lambda_{1}\right)=-2 \Delta ; \\
\mu\left(\omega_{2}, \lambda_{1}\right)=-\Delta ; \\
\mu\left(\omega_{2}, \lambda_{2}\right)=\Delta ; \\
\mu\left(\omega_{1}, \lambda_{2}\right)=2 \Delta .
\end{array}\right.
$$

Fig. 9 presents error rates of HMM-IN, mixture-HMM-IN and TMMIN estimators. The curves related to HMM-IN-2 and mixture-HMMIN-2 estimators were omitted as they were identical to those of HMM-IN and mixture-HMM-IN respectively. We observe that the non-mixture classic models are asymptotically suboptimal.

In Case 3 , we consider $\Delta>0$ and set

$$
\left\{\begin{array}{l}
\mu\left(\omega_{1}, \lambda_{1}\right)=-2 \Delta ; \\
\mu\left(\omega_{2}, \lambda_{1}\right)=-\Delta ; \\
\mu\left(\omega_{1}, \lambda_{2}\right)=\Delta ; \\
\mu\left(\omega_{2}, \lambda_{2}\right)=2 \Delta .
\end{array}\right.
$$




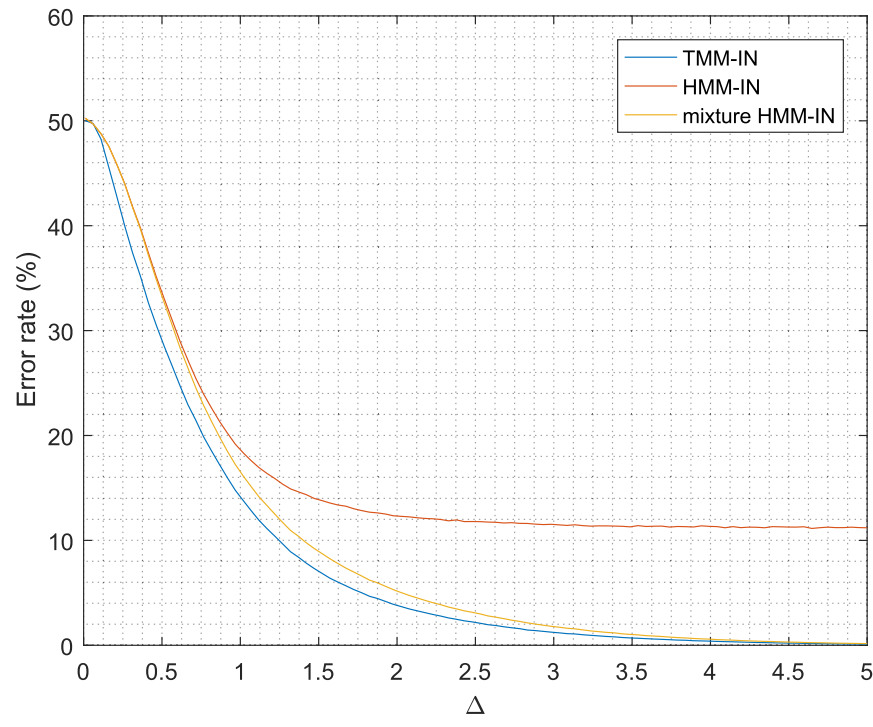

Fig. 9. Performances comparison between the TMM-IN estimator and its approximations given by the classic models in Case 2, for various $\Delta$ in (53). Sample size is 1000 and the results are averaged over 100 experiments.

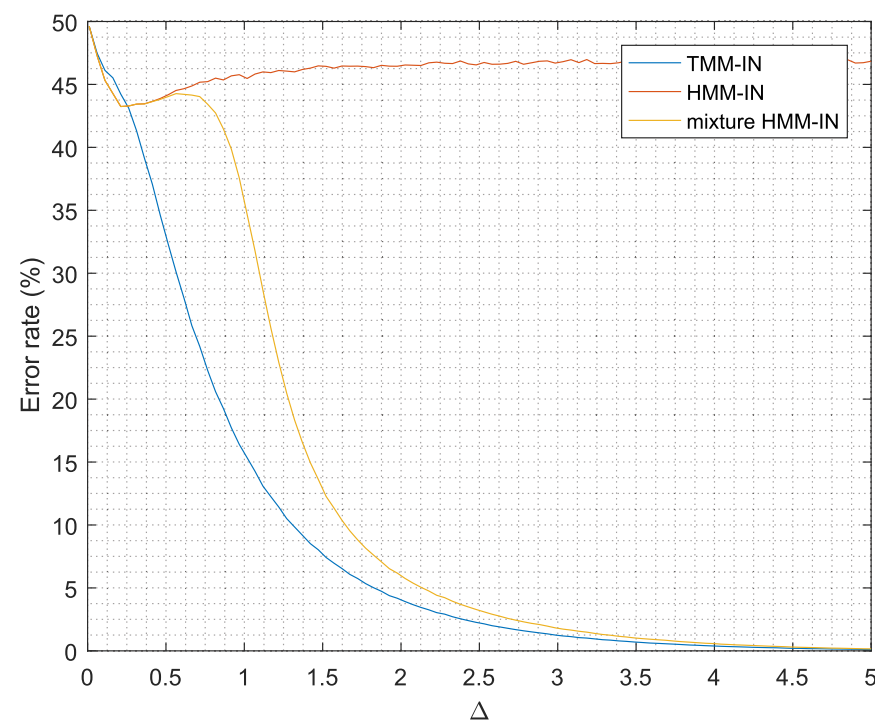

Fig. 10. Performances comparison between the TMM-IN estimator and its approximations given by the classic models in Case 3, for various $\Delta$ in (54). Sample size is 1000 and the results are averaged over 100 experiments.

Fig. 10 presents error rates of HMM-IN, mixture-HMM-IN and TMM-IN estimators in Case 3. The curves related to HMM-IN-2 and mixture-HMM-IN-2 estimators were omitted as they were identical to those of HMM-IN and mixture-HMM-IN respectively. We observe that the non-mixture classic models diverge. Moreover, we see that the TMM-IN estimator may be significantly more accurate than that of the classic models. The gap we observe between classic mixture-based and TMM-IN estimators is due to taking the Markovianity of $(\mathbf{X}, \mathbf{U})$ into account. We notice that extending the state space of classic models may not result in improving the accuracy of the corresponding estimators.

\section{Conclusions and perspectives}

The objective of this work was to compare the accuracy of Bayesian MPM estimators based on the classic hidden Markov model (HMM) and its extensions which are the pairwise Markov model (PMM) and the triplet Markov model (TMM). The main conclusion is that PMMs and TMMs may offer substantial improvements of the estimation accuracy when compared to HMMs. Such improvements were particularly visible when the observation distribution was heavily autocorrelated and/or if the hidden chain was far from being Markovian. This conclusion is based on experiments on synthetic and real-world data.

Let us mention the perspectives for further research. Firstly, PMMs and TMMs can be extended to handle fuzzy jumps $[25,26]$ and a similar study could be also considered on the same methodological basis. Next, the copula-based TMM would allow an advanced modeling dependencies in $p\left(\mathrm{y}_{1}, \mathrm{y}_{2} \mid \mathrm{u}_{1}, \mathrm{u}_{2}, \mathrm{x}_{1}\right.$, $\left.\mathrm{x}_{1}\right)$. Specifically, the choice of the copula for modeling $p\left(\mathrm{y}_{1}, \mathrm{y}_{2} \mid \mathrm{x}_{1}\right.$, $\mathrm{x}_{1}$ ) in models of type HMM-CN is relevant to the performance of the corresponding estimators $[27,28]$.

\section{References}

[1] O. Cappé, E. Moulines, T. Rydén, Inference in Hidden Markov Models, SpringerVerlag, 2005, doi:10.1007/0-387-28982-8

[2] Y. Ephraim, N. Merhav, Hidden Markov processes, IEEE Trans. Inf. Theory 48 (6) (2002) 1518-1569, doi:10.1109/TIT.2002.1003838

[3] L.R. Rabiner, A tutorial on hidden Markov models and selected applications in speech recognition, Proc. IEEE 77 (2) (1989) 257-286, doi:10.1109/5.18626.

[4] R. Bhar, S. Hamori, Hidden Markov Models: Applications to Financial Economics, vol. 40, Springer Science \& Business Media, 2006, doi:10.1007/ b109046.

[5] R.S. Mamon, R.J. Elliott, Hidden Markov Models in Finance, vol. 4, Springer, 2007, doi:10.1007/0-387-71163-5.

[6] T. Koski, Hidden Markov Models for Bioinformatics, vol. 2, Springer, Netherlands, 2001

[7] M. Vidyasagar, Hidden Markov Processes: Theory and Applications to Biology, Princeton University Press, 2014.

[8] W. Pieczynski, Pairwise Markov chains, IEEE Trans. Pattern Anal. Mach. Intell. 25 (5) (2003) 634-639, doi:10.1109/TPAMI.2003.1195998.

[9] P. Lanchantin, J. Lapuyade-Lahorgue, W. Pieczynski, Unsupervised segmentation of randomly switching data hidden with non-Gaussian correlated noise, Signal Process. 91 (2) (2011) 163-175, doi:10.1016/j.sigpro.2010.05.033.

[10] S. Derrode, W. Pieczynski, Signal and image segmentation using pairwise Markov chains, IEEE Trans. Signal Process. 52 (9) (2004) 2477-2489, doi:10. 1109/TSP.2004.832015.

[11] J. Lember, H. Matzinger, J. Sova, F. Zucca, Lower bounds for moments of global scores of pairwise Markov chains, Stoch. Process. Their Appl. (2016). to appear

[12] W. Pieczynski, C. Hulard, T. Veit, Triplet Markov Chains in Hidden Signal Restoration, 4885, Crete, Greece, 2003, pp. 58-68, doi:10.1117/12.463183.

[13] J. Lapuyade-Lahorgue, W. Pieczynski, Unsupervised segmentation of hidden semi-Markov non-stationary chains, Signal Process. 92 (1) (2012) 29-42. https: //doi.org/10.1016/j.sigpro.2011.06.001.

[14] S.-Z. Yu (Ed.), Hidden Semi-Markov Models, Elsevier, Boston, 2016, doi:10.1016/ B978-0-12-802767-7.00010-3.

[15] V.S. Barbu, N. Limnios (Eds.), Semi-Markov Chains and Hidden Semi-Markov Models Toward Applications, Springer-Verlag, New York, 2016, doi:10.1007/ 978-0-387-73173-5.

[16] Y. Ephraim, B.L. Mark, Causal recursive parameter estimation for discrete-time hidden bivariate Markov chains., IEEE Trans. Signal Processing 63 (8) (2015) 2108-2117, doi:10.1109/TSP.2015.2408557.

[17] Y. Sun, B.L. Mark, Y. Ephraim, Collaborative spectrum sensing via online estimation of hidden bivariate Markov models, IEEE Trans. Wireless Commun. 15 (8) (2016) 5430-5439, doi:10.1109/TWC.2016.2558506.

[18] S.L. Cam, F. Salzenstein, C. Collet, Fuzzy pairwise Markov chain to segment correlated noisy data, Signal Process. 88 (10) (2008) 2526-2541. https://doi.org/10. 1016/j.sigpro.2008.05.003.

[19] S. Bricq, C. Collet, J.P. Armspach, Triplet Markov chain for 3D MRI brain segmentation using a probabilistic atlas, in: 3rd IEEE International Symposium on Biomedical Imaging: Nano to Macro, 2006, 2006, pp. 386-389, doi:10.1109/ ISBI.2006.1624934

[20] C. Sammut, G. Webb, Encyclopedia of Machine Learning, Springer US, 2011, doi:10.1007/978-0-387-30164-8.

[21] I. Gorynin, E. Monfrini, W. Pieczynski, Pairwise Markov models for stock index forecasting, in: 2017 25rd European Signal Processing Conference (EUSIPCO), Greece, Kos Island, 2017, pp. 2095-2099.

[22] N. Lethanh, B.T. Adey, Use of exponential hidden Markov models for modelling pavement deterioration, Int. J. Pavement Eng. 14 (7) (2013) 645-654, doi:10. 1080/10298436.2012.715647.

[23] C. Gourieroux, J. Jasiak, Autoregressive gamma processes, J. Forecasting 25 (2) (2006) 129-152, doi:10.1002/for.978.

[24] D.B. Paul, The Lincoln tied-mixture HMM continuous speech recognizer, in: Acoustics, Speech, and Signal Processing, 1991. ICASSP-91, 1991, International Conference on, IEEE, 1991, pp. 329-332, doi:10.1109/ICASSP.1991.150343. 
[25] C. Carincotte, S. Derrode, S. Bourennane, Unsupervised change detection on SAR images using fuzzy hidden Markov chains, IEEE Trans. Geosci. Remote Sens. 44 (2) (2006) 432-441, doi:10.1109/TGRS.2005.861007.

[26] F. Salzenstein, C. Collet, S. Lecam, M. Hatt, Non-stationary fuzzy Markov chain, Pattern Recogn. Lett. 28 (16) (2007) 2201-2208, doi:10.1016/j.patrec.2007.07. 002.
[27] N. Brunel, W. Pieczynski, Unsupervised signal restoration using hidden Markov chains with copulas, Signal Process. 85 (12) (2005) 2304-2315, doi:10.1016/j. sigpro.2005.01.018.

[28] S. Derrode, W. Pieczynski, Unsupervised classification using hidden Markov chain with unknown noise copulas and margins, Signal Process. 128 (2016) 8-17, doi:10.1016/j.sigpro.2016.03.008. 